\section{Iatrogenic Ulcers of the Small Intestine}

SIR,-We should like to report another possible case of ileal ulceration and perforation in a patient receiving enteric-coated potassium-chloride tablets but not chlorothiazide.

In June, 1964, a woman aged 58 was diagnosed as having pemphigus vulgaris. This proved difficult to control and necessitated an initial suppressive dose of $210 \mathrm{mg}$. prednisolone and 120 units corticotrophin gel daily. Control was achieved and the steroids were gradually withdrawn during the following four months without recurrence of the permphigus. Her serum potassium fell steadily during treatment despite constant administration of enteric-coated potassium-chloride tablets $1 \mathrm{~g}$. twice daily increasing on 19 August to $2 \mathrm{~g}$. three times a day. It was suspected, and later confirmed, that she was not taking all her potassium and she discharged herself on 26 August with a serum potassium of only $2.7 \mathrm{mEq} / 1$. She was warned of the possible consequences and by 2 September her potassium was 5.1 rising to $5.5 \mathrm{mEq} / 1$., suggesting that she was now taking the full dose of potassium chloride-that is $6 \mathrm{~g}$. daily.

At about this time she began to complain of vague lower abdominal pain associated with tenderness and diarrhoea. Her E.S.R., which had varied from 25 to $45 \mathrm{~mm}$. (Westergren), rose to $85 \mathrm{~mm}$./hr. and has since fluctuated between 44 and $116 \mathrm{~mm}$. On 1 November the pulse rate rose suddenly to 120 and she was mildly shocked. A gastrointestinal perforation was suspected and she consented to admission but refused operation. The haemoglobin fell to $58 \%$ and occult bloods were positive. Triamcinolone was finally discontinued on 22 November but potassium chloride was continued. She gradually improved until on 10 December 1964 a barium enema revealed a fistula between the ileum and pelvic colon. A few diverticula were present in the colon. At no time during her illness were thiazide-type diuretics administered. -We are, etc.,

\section{K. D. Crow.}

A. G. FREEMAN

G. C. Griffiths.

J. A. M. AGRR.

E. E. Evans.

Princess Margaret Hospital,

Swindon, Wilts.

\section{Rapid Estimation of Blood Glucose}

SiR,-Like Dr. Vincent Marks and Mr. A. Dawson (30 January, p. 293) we have been satisfied with Dextrostix when used for bedside estimations of blood glucose on diabetic children undergoing stabilization. When this technique is used on the newborn, however, there are important qualifications, as the following case history illustrates:

A female infant, mature by date, weighed $5 \mathrm{lb} .12 \mathrm{oz}$. at birth which was rapid and unassisted. She cried at once and accepted small feeds (sweetened water followed by dilute milk) during the first 48 hours. At 48 hours she had a sudden prolnnged cyanotic attack and was rushed to hospital. On arrival she was deeply cyanosed and jactitating, but was successfully resuscitated. A blood-sugar estimation was carried out using a heel-prick sample by the Dextrostix method. The foot was cyanosed and cool, but bled freely: the blood appeared dark and concentrated. No colour change occurred on the Dextrostix paper, indicating blood-glucose concentration of less than $40 \mathrm{mg}$.I $100 \mathrm{ml}$. The umbilical vein was catheterized at once and a venous sample obtained: an infusion of $25 \%$ glucose solution was then given. By the Folin and $\mathbf{W u}$ method the venous bloodsugar level before the infusion was found to have been $80 \mathrm{mg} . / 100 \mathrm{ml}$. Meanwhile a further infusion of $20 \%$ fructose snlution had been given. After 15 minutes the Dextrostix test was again applied to blond from annther puncture of the same heel; again no colour change was observed, using strips from two separate boxes. Immediately afterwards blood drawn from the umbilical catheter gave the maximum colour change on a Dextrostix, and by the Folin and Wu method a value of $800 \mathrm{mg} . / 100 \mathrm{ml}$. was obtained.

We conclude that when there is stagnation of the peripheral circulation, as in the cyanosed limbs of an ailing neonate, the glucose level in heel-prick blood may fall to a level quite unrepresentative of that in the central circulation. Therefore, to rely on heel-prick samples for the diagnosis of hypoglycaemia in neonates may prove seriously misleading.-We are, etc.,

\section{Malcolm MacGregor.} RONALD ROBINSON.

Warwick Hospital, Warwick.

\section{Ischaemic Heart Disease and Pregnancy}

SIR,-Dr. M. F. Oliver in his letter (30 January, p. 315) on oral contraceptives and coronary thrombosis states that "from all the millions of pregnancies only some 30 cases of ischaemic heart disease have now been recorded." Although the incidence of coronary-artery disease associated with pregnancy would seem to be very low, Mendelson ${ }^{1}$ lists 45 cases and refers to another 12 instances of anginal syndrome. Seventeen of the 45 were reported in the five years up to 1959 , perhaps due to an increased certainty in modern diagnostic methods. Further cases have been reported since and Pfaffenschlager ${ }^{2}$ reported a case of myocardial infarction in a 21-year-old gravida.

I am grateful to Mr. F. Selby Tait for permission to report a further case of effort angina in pregnancy which was recently under his care.

The patient aged 41 years, para $3+0$, a suillbirth at 30 weeks in 1949, and normal deliveries at term in 1950 and $1952(3,380 \mathrm{~g}$. and 3,235 g.), was first seen in September 1963, ten weeks after her last menstrual period with a history of angina of effort. For two months she had experienced precordial pain on walking, which forced her to stop and was relieved by rest, but recurred on further exertion. There was no history or signs of previous rheumatic fever. The weight was $69 \mathrm{~kg}$., the blood-pressure 130/70 $\mathrm{mm}$. of mercury, the haemoglobin concentration 12.7 g. $/ 100 \mathrm{ml}$., and the Wassermann reaction negative. The resting electrocardiograph showed confirmatory changes in the S.T. segment; the radiographic cardiovascular silhouette was within normal limits.

The pregnancy progressed normally, the anginal symptoms, which persisted only until the 25 th week, were relieved by glyceryl trinitrate. The blood-pressure ranged between $90 / 60$ and $130 / 70 \mathrm{~mm}$. of mercury. There was no oedema and the total weight gain was $10.4 \mathrm{~kg}$.

She was admitted to hospital on 1 May 1964, two days after the expected date of confinement. The onset of labour was spontaneous six days later. After a first stage of $3 \frac{1}{3}$ hours, and 30 minutes in the second stage, she was delivered as a prophylaxis by low-forceps extraction under pudendal-block anaesthesia of a healthy living female infant weighing $3,725 \mathrm{~g}$. The third stage was normal. Lactation was suppressed. She was discharged home after an uneventful early puerperium on the ninth day. When she attended the post-natal clinic six weeks postpartum she was tired and said she had experienced further chest pain. She was referred for family-planning advice.
While agreeing that ischaemic heart disease is uncommon in pregnancy $I$ feel that there must be other unrecorded cases and that this serves to emphasize the rarity.-I am, etc.,

H. Oliphant Nicholson.

Queen Elizabeth Hospital Birmingham.

\section{REPERENCES}

1 Mendelson, C. L., Cardiac Disease in Pregnancy. 1960. F. Davis, Philadelphia.

Pfaffenschlager, F., Wien. Klin. Wschr., 1964, $76,297$.

\section{Oral Contraceptives and Breast Cancer}

SiR,-Dr. B. A. Stoll (3 October, p. 875) in a reply to the letter of Mr. J. J. Shipman (5 September, p. 629) writes that the experimental studies of Huggins stand against Shipman's supposition that the commonly used oral contraceptives may predispose toward the development of breast cancer or accelerate its growth when present.

Huggins $^{2}$ used massive doses of progesterone and oestrogen and found the efficacy of his regimen dependent on a certain ratio of oestradiol-17 $\beta$ to progesterone $(20 \mathrm{mg}$. of the former to $4 \mu \mathrm{g}$. of the latter daily for 30 days). In the first place it cannot be said that the relative doses of progesterone and oestrogen in the oral contraceptive preparations are similarly balanced, nor do they approach the massive level required for demonstration of the effect in Huggins's study. Furthermore, it has been shown clearly that norethindrone and norethynodrel have a strong inherent oestrogenicity. At least in the case of those oral contraceptive agents including these compounds as the progestational component the balance of progesterone versus oestrogen action may strongly favour oestrogenic effects. ${ }^{2}$ The importance of dose levels in determining the response of metastatic breast-cancer growth in post-menopausal women is critical. Low doses may accelerate, large doses suppress.

Finally, it is clearly undesirable for medical men to equate the physiologic actions of the synthetic 19 nor-steroids and the various substituted pregnane derivatives with those of progesterone itself. Semantic sins of this sort are apt to extort a rather high cost against accuracy in thought, interpretation, and therapeutic planning.-I am, etc.,

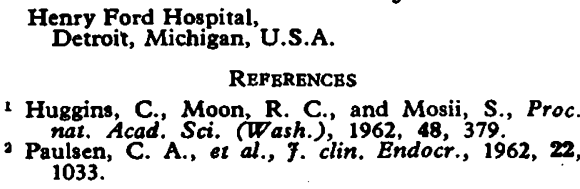
Henry Ford Hospital, REFBRENCBS

1 Huggins, C., Moon, R. C., and Mosii, S., Proc Paulsen, C. A., et al., J.) clin. Endocr., 1962, 22 1033. Michael J. Brennan.

\section{Oral Contraceptives and Coronary Thrombosis}

SIR,-Dr. J. H. Naysmith (23 January, p. 250) asks if there is an increased risk of coronary thrombosis occurring in women taking the pill, and if so are we justified in subjecting our patients to this risk. In the case which he describes Dr. Naysmith does not state whether necropsy revealed atheromatous change in the coronary arteries. Dr. Flora M. Hartveit (2 January, p. 60) describes an incident of fatal coronary thrombosis in a woman taking oral contraceptives and where detailed necropsy failed to show the presence 
of atheromatous change in the coronary arteries. This latter observation could well be found significant in a prospective study to relate the use of oral contraceptives and coronary thrombosis.

Oral contraceptives may contain both oestrogens and progestogens. The effects of each should be considered.

Premature withdrawal of natural oestrogens has been shown to increase as much as sevenfold the incidence of coronary thrombosis. ${ }^{1}$ : Stander has demonstrated that there is a significant increase in serum cholesterol in women who have been castrated or who experience an early menopause. Davis ${ }^{3}$ has further demonstrated that oestrogenreplacement therapy reduces the degree and rate of increase of serum cholesterol in such women. Current evidence suggests that oestrogens will reduce rather than increase the risk of coronary thrombosis.

There is also some evidence of the possible effect of progesterone or progestogens on the coronary arteries. In 1960 Watson and his colleagues ${ }^{4}$ were able to present 24 cases collected from the literature of coronary thrombosis occurring in young pregnant women and added two cases of their own. These are very small numbers and suggest that the incidence of coronary thrombosis is rare compared with venous thrombosis in pregnancy. There is evidence ${ }^{5}$ that progestogen therapy is associated with an increase in some coagulation factors which could increase the clotting tendency. There is no information, as far as I am aware, to relate progestogens alone with coronary thrombosis, though these hormones have been used for some years.

On the whole I feel that Dr. Naysmith could be advised that there is at present insufficient evidence to show that oral contraceptives increase the risk of coronary thrombosis. The relationship could as easily be casual as causal, and the use of oral contraceptives should not be withheld on the grounds of increasing the risk of coronary thrombosis.-I am, etc.,

Hull, Yorks.

\section{GARETH LlOYd.}

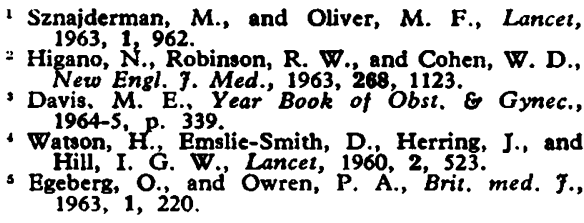

\section{"Epidemic Collapse"}

SIR,-I read with interest the article by Drs. G. T. Pollock and T. Morrison Clayton (26 December, p. 1625), and the suggestion by Dr. A. Frame ( 9 January, p. 127) that the symptoms could be attributed to the syncope following modified Valsalva mancuvres.

I consider the outbreak unlikely to be due to Valsalva manœuvres. During a misspent boyhood I regret to admit that my young colleagues and I engaged in this experiment. Loss of consciousness usually supervened, occasionally accompanied by convulsions. Recovery was usually complete within minutes. Vomiting and upper abdomina pain did not usually occur, and the feeling of faintness did not persist. These symptoms were prominent in the outbreak so carefully described by Drs. Pollock and Morrison
Clayton, and persisted for some while in children, who presumably were kept under continuous observation. If one recalls the applied physiology of a modified Valsalva's manœuvre, one will find it unlikely for resulting symptoms to persist for so long afterwards.

I suggest, therefore, that the outbreak must be attributed to a virus infection or possibly an unknown toxin. The latter may even have been self-administered.-I am, etc.,

$$
\underset{\text { Essex. }}{\text { Canvey Island, }} \text { P. G. HAWKer. }
$$

\section{New Type of Accident}

Sir,-Dr. Alan J. Byron's letter (New Type of Accident) (16 January, p. 192) prompts me to report a similar case seen in the casualty department here some time back.

The patient was a boy of 17, an apprentice mechanic at a local factory, and he was working with an electric hand-drill which had a built-in fan to cool the system. After working for a while he felt very hot, and started perspiring, and decided to cool his forehead with the fan in the drill, which he held near his forehead. In so doing he caught a lock of his front curls in the fan and jerked his head away from it. A wedge of hair, 2 in. by 2 in. $(5 \mathrm{~cm}$. by $5 \mathrm{~cm}$.), was avulsed but no scalp removed. The wound was dressed and it healed satisfactorily in due course.

Whilst I quite agree that the legislation regarding wearing protective hair nets should be extended to men as well, I wonder if it is only the length of hair, which was normal in this case, or whether it is the ignorance of the hazards of fast machinery amongst the apprentices that matters as well.

My thanks are due to Mr. F. W. T. Davies, F.R.C.S., Orthopaedic Surgeon, for permission to publish this case.

-I am, etc.,

The General Hospital,
Burton on Trent,

M. J. SHAH. Burton on
Stafis.

\section{Idiopathic Splenomegaly}

SIR,-The paper by Dr. P. D. Marsden and his colleagues (9 January, p. 89) describing a valuable investigation on tropical or idiopathic splenomegaly in Uganda drew attention to the possible causative role of long-standing infections with Plasmodium malariae as shown by the more frequent finding of parasites in their patients when compared with " controls" and also by the results of fluorescent-antibody tests in the two groups. The authors wisely stated that the relationship between splenomegaly, lymphocytic infiltration of the hepatic sinusoids, and quartan malaria should be regarded as a working hypothesis. Two points raised in this excellent paper should be stressed, as they need further investigation.

First, that in tropical Africa the term "splenomegaly" should be always related to the age of the patient or of the examined group. Moreover, when referring to adult Africans living in a malarious area "splenomegaly" must be understood in its relative, clinical meaning - namely, the presence of an unusually large and palpable spleen. This "petitio principii" is not as absurd as it sounds !
Based on the traditional simple spleenpalpation carried out in the standing or recumbent position the "spleen census" of a malaria survey of the unselected indigenous population of tropical Africa shows usually the classical results of a high (over $75 \%$ ) spleen rate in children and a low (about 15\%) spleen rate in adults. A biometric study of spleen weights based on 4,560 necropsies in Lagos, Nigeria, ${ }^{1}$ showed that the apparently low frequency of enlarged spleens found on palpation of the adult Africans living in a malarious area is deceptive. In fact at least $80 \%$ of these "not-palpable" adult spleens are enlarged to nearly twice the "normal" size, since their average weight is 265 against $160-170 \mathrm{~g}$. of the non-African spleen. The epidemiological conclusions of this investigation are not relevant for the purpose of the present letter, but I suggest that more biometric studies of the data obtained from the routine work of morbid anatomy should be carried out in teaching hospitals and departments of pathology in Africa.

The second point refers to Marsden's and his colleagues' conclusion that infections with $P$. malariae in Africa are much more common than it was thought previously. This is true not only for a selected sample from a hospital but also for a larger, unselected sample of overall population-especially in rural areas of Africa. It has been shown in Nigeria that careful and repeated blood examinations carried out during 2 longitudinal survey of asymptomatic adult carriers of malaria parasites will reveal that the true rate of $P$. malariae infections is $20 \%$ as compared to about $2 \%$ of the single blood examination."

Apart from its importance for our better understanding of the natural history of holoendemic malaria this finding indicates the inadequacy of our present routine methods for reliable detection of -malaria parasites when the parasites are very scanty.

It is not improbable that further development of new immunological and other methods may be of much help in this field. But that is another story $1-\mathrm{I}$ am, etc.,

World Health Organization

L. J. Bruce-Chwatt.

Geneva, Switzerland.

\section{REFERENCES}

1 Bruce-Chwatt, L. J., Bull. Wld Hlth Org., 1956. 15, 513. Afr. med. J., 1963, 12, 141 and 199.

\section{Behçet's Syndrome}

SIR,-I would like to make a comment about the Clinicopathological Conference (6 February, p. 357). The discussion concerning the occurrence of sinus thrombosis in relation to Behçet's syndrome attracted my attention, since I reported a similar case to the Clinical Section of the Royal Society of Medicine in $1959 .{ }^{\prime}$

The case that I demonstrated was a man aged 29 , and sinus thrombosis had occurred one week after a moderately intensive 18-day course of prednisone. The difficulty was that there might have been hypercoagulability of the blood after the cortisone-analogue therapy, ${ }^{2}$ which could have accounted for the event. The case described in the Clinicopathological Conference had no prednisone until 16 months after the episode of duralsinus thrombosis, so that it must be diseaserelated. 